\title{
SENSOR DATA FUSION THROUGH A DISTRIBUTED BLACKBOARD
}

\author{
S. Y. Harmon, G. L. Bianchini, B. E. Pinz
}

Code 442, Naval Ocean System Center San Diego, CA, 92152

\section{ABSTRACT}

Sensor data fusion takes many forms and has diverse purposes. Sensor data fusion can improve a complex robot's picture of the world and increase its confidence in the truth of that picture. A computing architecture which supports many different schemes for fusing sensor data is necessary to support the activities of complex robots. One such architecture is the distributed blackboard mechanism implemented onboard the USMC Ground Survejllance Robot (GSR) [1]. The distributed blackboard has proven to be a very useful and flexible mechanism through which to accomplish effective sensor data fusion.

\section{INTRODUCTION}

Today, multiple, redundant and disparate sensors are abundant. Many existing complex mobile robots employ multiple sensors (e.g. TV cameras, acoustic, infrared and laser rangefinders, absolute and relative navigation sensors, force and tactile sensors, etc.). As the capabilities of complex robots increase they can undertake tasks of greater complexity and perform more robustly in uncertain and unknown task environments. Sensor data fusion is a logical step towards increasing robot capability. It lets a robot make best use of all of its sensor resources to solve complex tasks. Sensor data fusion can improve a coinplex robot's performance by providing hybrid information, by reducing sensor errors and by maximizing sensor use.

Hybrid information is information derived from the combination of sensor data which would not be available from a single sensor source. It can represent new physical properties about known objects in the task environment $[1,2]$ or identification of objects previously unknown to the robot $[3,4]$. Allen fuses stereo vision and active tactile sensing data for object recognition [3]. Data fusion can reduce sensor errors generated by inaccurate sensor data interpretation mode 1s, by sensors driven beyond their bounds of known accuracy and by sensor failures. Flynn investigated the use of multiple sensors with complementary characteristics to reduce the error inherent in sensing mechanisms [5]. Her system also exploited sensor redundancy to recover from sensor failure. The multiplicity of data available from redundant sensors is a useful feature which can go under utilized if a coherent methodology is not developed to analyze sensor performance. This analysis will almost always involve the fusion of data from many sensors. Sensor use can be maximized by using data fusion to optimally control sensor application, to aid in sensor calibration and to filter noisy sensor data. Kent uses fast infrared proximity detectors and a video camera to grasp dynamic objects [4]. In his system the vision system generates realtime calibration parameters for the proximity system. Brooks [6] and Iberall [7] have developed methods for hiding sensory data by representing it in a filtered form which depends upon the perceptive processing needs. Perceptive processing needs can also guide the control of sensors in data gathering activities [7]. In another example, the Ground Surveillance Robot (GSR) uses steered acoustic rangefinders and a vision system mounted on a three degree of freedom platform for target tracking [1]. Each sensor system relays target bearing information to the other to help servo its field of view in the proper direction.

\section{SENSOR DATA FUSION TECHNIQUE}

In the simplest situation, a robot with multiple sensors uses them independently. As the data from multiple sensors are merged the coupling complexity increases the design complexity significantly. The first step toward dealing with this complexity is to use a uniform sensor data representation. This agreement in representation is necessary to permit economical communication between distributed sensor subsystems. Data from like sensors are the simplest to fuse since they have the same dynamic responses, update rates, calibration and error conditions. Often like sensor data can be most effectively fused at the lowest levels of the computing hierarchy. Data fusion problems increase further when the information from disparate sensors is merged. If the physical domains of disparate sensors intersect then data fusion requires knowledge of the observation times, the sensor responses (both static calibration and error) and the fusion method (i.e., what model is used to equate two different measurements of the same phenomenon). Several methods can be used to fuse disparate sensor data. of course, a representation and a 
collection of methods are not sufficient for practical sensor data fusion. Timely effective data fusion requires a flexible computing architecture. The architecture discussed in this paper uses a distributed blackboard paradigm. Several examples of sensor data fusion onboard the GSR are discussed to iliustrate the power of the distributed blackboard technique.

\section{Representation}

A robot task environment $E$ can be modelled as a finite unordered set of objects, 0 , such that

$$
E=\left(0_{1}, 0, \cdots, 0\right)
$$

Each object 0 in this set has an associated set of properties $P$ where

$$
P_{i}=\left(P_{i, 1}, P_{i, 2}, \cdots, P_{i, m}\right)
$$

And each object property has a single value hence the simplest task environment representation is the set of 3-tuples

$$
\begin{gathered}
E=\left(\left(0_{1}, P_{1,1}, v\right),\right. \\
\vdots \\
\left.\left(0_{n}, P_{n, m}, v\right)\right)
\end{gathered}
$$

Unfortunately, knowledge of the environment can not be obtained directly but must be measured through sensors plagued by the inaccuracies of the real world. Sensors introduce errors for a variety of reasons including inherent uncertainties, discrete sampling and quantification errors, inaccurate calibration and device failures. If no device failures have occurred a sensor's accuracy can usually be calculated from empirical and analytic sources. If a sensor's accuracy is dependent upon information from other sensors its computation can involve data fusion. Often good estimates of a sensor's accuracy made in a controlled environment can aid sensor performance analysis in unknown environments. Most error recovery systems proposed for robots depend at least in part on prior knowledge of the task environment [8]. However, many mobile robot applications make this assumption impractical $[1,9]$. Dynamic estimation of sensor accuracy through fusion of data from redundant sensors may permit identification of sensor failure in complex unknown environments. For these reasons and others, estimates of property value accuracies are necessary for fusion of real world estimates of object property values. Thus, each sensor obtained value for which an accuracy estimate is available adds an additional value e (for estimated error) to the objectproperty tuple. This changes the task environment representation to

$$
\begin{array}{r}
E=\left(\left(o_{1}, p_{1,1},(v, e)\right),\right. \\
\left.\quad\left(0_{n}, p_{n, m},(v, e)\right)\right)
\end{array}
$$

and $e=$ Function-of $(r)$ where $r=$ subset-of(E)

Another notion useful to this data representation is the confidence in the interpretation of a property's value. Confidence measures have been proposed as a primary means for fusing data about the same object properties from different sensors $[2,3]$. Usually measures of confidence represent the sensor source's faith in the model it uses to interpret property values and error estimates. Most simply, a confidence measure can be used as the criterion to choose between conflicting sensor estimates of the same property value. It can also be used for setting thresholds and weights in various averaging schemes. In this scheme, confidence measures are assigned at the moment the information is made available to other consumers. As a result, these measures can become inaccurate in dynamic situations. Consistent confidence measure scaling is critical if confidence is used in sensor data fusion. Nevertheless, confidence, $c$, is a key aspect of sensor data representation thus changing the object-property tuples in the task environment representation to

$$
\begin{array}{r}
E=\left(\left(0, p_{1,1},(v, e, c)\right),\right. \\
\left.\left.\quad \underset{n}{\left(0, p_{n, m}\right.},(v, e, c)\right)\right)
\end{array}
$$

Unfortunately, (3) remains an inadequate representation in most task domains because it takes no account of the changing property values of a dynamic world and, fails to provide any basis for temporal reasoning. A static representation of unchanging or slowly changing (relative to the length of the task) properties is sufficient. However, many properties change too rapidiy to ignore temporal considerations. often a significant amount of time passes between the time a measurement was made by a sensor and the time it is used. Knowledge of this time interval is necessary for data fusion in dynamic situations so decisions can be made as to how best to use the information and so estimates of current values can be predicted (provided the nature of the dynamics are known). In a distributed computing environment allowing access to different sensor data on multiple occasions by different consumers (e.g., other sensor modules, controllers and planners) decouples complex processing and lowers communication needs. The knowledge of the time elapsed since property measurement can be recorded as the observation timestamp made on some absolute time scale (usually defined by a system clock although this function can also be distributed between several computing modules if some synchronization scheme is used). Therefore, complete task environment representation must include the observation timestamp, $t$, with each 
object-property tuple

$$
\begin{aligned}
E= & \left(\left(0_{1,}, p_{1,1},(v, e, c, t)\right),\right. \\
& \left.\left(0_{n}, p_{n, m} ;(v, e, c, t)\right)\right)
\end{aligned}
$$

Some error is to be expected in the timestamp itself due to inaccurate synchronization, delays introduced by the sensor and any processing overhead in acquiring the data and associating the timestamp. Timestamping sensor data should take place as near to actual data acquisition as possible and known delays should be taken into account in order to minimize these errors. occasionally, calculation of the sensing delay is dependent upon the present state of the task environment and, thus, requires knowledge of other sensed values. These cases represent other examples when sensor data fusion can increase the precision of property value estimates.

\section{Methods}

Several methods exist for fusing sensor data depending upon the situation. The simplest situations represent complementary sensor measurements. If two sensor observations, $A$ and $B$, are complementary and describe similar (i.e., same measurement units) but independent situations (i.e., no intersection between measurements) then they can be logically added to the total environment description without concern for conflict. Each measurement also contributes its own associated values for accuracy and confidence. If these observations are complementary but coupled (i.e., $C=f(A, B)$ ) then the dependent quantity can be computed from the prevailing model (i.e., $f()$ ) and input measurements (i.e., $A$ and B). The dependent quantity's accuracy and confidence can also be computed from this model.

More complex situations arise when sensor
observations intersect information about the same property value of the same object) In these situations the information must be merged. If intersecting measurements represent observations at different times or at different but continuous spatial positions then simple interpolation is the most straightforward merging method. Accuracies and confidences associated with interpolated values can also be interpolated although more sophisticated models for computing these parameters can be used if necessary. Several merging methods are available if independent sensor observations are completely intersecting (i.e., same object, same property, same spatial location and approximately the same time) including some form of averaging, a decision between two intersecting sensor observations and the act of guiding the processing of one observation with another.

\section{Averaging}

This method, along with guiding method mentioned below, uses confidence measures in the spirit that they are usually intended $[2,3]$. This is a simple percentage calculation based on confidences which are then used as weights in an averaging of sensor data values. Given readings ( $V 1, V 2, \ldots, V n)$ and the associated confidences ( $\mathrm{Cl}, \mathrm{C} 2, \ldots, \mathrm{Cn}$ ) from sensors $S 1, S 2, \ldots, S n$ then averaging weights can be computed by

$$
w_{i}=C_{i} /\left(C_{1}+C_{2}+\ldots+C_{n}\right)
$$

and the fused estimate becomes

$$
v_{f}=w_{1} * v_{1}+w_{2} * v_{2}+\ldots+w_{n} * v_{n}
$$

The confidence in the fused value can be most simply calculated from analogous equations

$$
w_{n}=v_{f} /\left(V_{1}+V_{2}+\ldots+V_{n}\right)
$$

and the fused confidence becomes

$$
C_{f}=W_{1} * C_{1}+W_{2} * C_{2}+\ldots+W_{n} * C_{n}
$$

Other more complex methods for computing confidence have been proposed including using error manifolds [10], heuristics [11] and probability theory [12]. The fused timestamp can be computed in many different ways. The latest or earliest timestamps could be chosen, a cluster of timestamps could be interpolated or the mean could be computed.

\section{Deciding}

This fusion method simply makes a discrete choice between several different measurements of the same property. This choice must be based upon some method to priority order the different measurements. The confidence associated with each value in the representation discussed above provides one means to make this ordering. In the simplest situation, the value with the highest confidence is chosen. However, more sophisticated heuristics can be used which may depend upon knowledge of the state of other parts of the task environment. In general, the confidence and timestamps associated with the fused values are those originally associated with those values. However, in many situations, Bayes rule can be used to compute the resultant confidences of fused values.

\section{Guiding}

In these fusion methods, the values from one sensor are used to guide the control or processing of other more accurate estimates from another sensor [4]. As implied, coarser estimates guide the definition of more accurate estimates. Guiding the thresholding of sensor input is one example of guiding the processing of sensor data. Thresholding is usually applied in combination with another method such as averaging to filter either the inputs or the output of the fusion process. A threshold value can be obtained either 
from an a priori constant, a previous sensor value or confidence or the resultant value or confidence of a fusion process. Thresholding the fusion output effectively filters multiple sensor readings and may be used as a measure of sensor performance $[2,3]$. Guiding can also apply to such processes as windowing to decrease search and steering controllable directional sensors (such as rangefinders and vision sensors with restricted fields of view).

\section{Implementation}

To this point, we have discussed a data representation and a collection of data fusion methods. These are just a collection of tools by which to accomplish sensor data fusion. In the following section, we discuss the application of those tools to the problem to sensor data fusion for an autonomous mobile robot, the GSR. The computing resources architecture on the GSR is configured to support data fusion at several different levels while minimizing consumption of costly internal communications bandwidth. Each subsystem shares sensor data through a blackboard mechanism which is implemented through interfaces to each module passing messages to transfer control and maintain blackboard consistency. Finally, examples of sensor data fusion using this technique on the GSR are discussed.

\section{Architecture}

In the distributed computing model currently employed, processing is distributed among subsystems of three types: sensor, control and knowledge based subsystems [13]. Subsystems are designed to be as capable and independent as possible. Communication between subsystems occurs at a high level and takes place through a local area network (LAN) (i.e.., IEEE 802.3). Processors within a subsystem communicate through shared memory accessed through a high speed parallel bus (i.e., IEEE 796). Sensor subsystems perform all the processing on sensor data to make symbolic information available to other subsystems through broadcast reports. Control subsystems provide all the processing needed to translate symbolic plans and sensor input into coordinated effector actions. Knowledge based planning subsystems use symbolic reports from sensor and control subsystems to formulate and distribute symbolic plans to the various subsystems.

Each subsystem's world model is defined by and designed into its own blackboard which resides in part of the subsystem's shared memory. Subsystems communicate through the Intelligent Communications Interfaces (ICIS) over the LAN. In this architecture, sensor data fusion can be done within a single processor (usually representing a single sensor group), between processors within a subsystem (usually closely interacting sensor and control groups) through the subsystem's blackboard memory and between subsystems (usually loosely coupled subsystems) through ICIs.
Distributed Blackboard Design

The blackboard is organized as a class tree data structure with inheritance properties and active functions. The blackboard actually resides in a subsystem's shared memory and is replicated in each subsystem. Class inheritance properties enable an economy of representation and generalization. classes are organized hierarchically with instances assigned to terminal classes. The class tree provides one way to relate blackboard objects to one another. Instances in the blackboard are represented, as discussed above, in object, property, value, confidence, timestamp tuples. Accuracy measures have not as yet been implemented although they are felt necessary for a complete data fusion capability. The timestamp attribute may be either generated automatically by the host operating system from the synchronized system clock or determined explicitly by the writing task. The confidence attribute is set based on the probability of error in a given instance measurement or calculation. Both the timestamp and confidence level are presently used to fuse data from multiple sources. Active functions provide data driven programming. access to the blackboard information and provide another way to relate one object to another. Standard blackboard interface procedures provide well defined access to the blackboard information in any processor or ICI in a subsystem.

\section{Intelligent Communications Interface}

The ICI mechanism defines a set of message passing protocols. The messages are communicated through the network and contain source identifier, priority, and body. The source address identifies the subsystem transmitting the message. The body may be a plan or a report. Plans can be either control plans or report plans. Control plans dictate how a subsystem should control some action. A report plan describes how a subsystem should generate reports on parts of its world mode?. A report communicates either plan status or information about some portion of the transmitting subsystem's world model. An ICI interprets incoming reports, updates the local blackboard with the new information and activates any active functions dependent upon recent blackboard state changes. A plan parser within the ICI parses plans received and makes new entries in report or control lists maintained in the blackboard. The ICI also implements a pattern matching function to recognize blackboard conditions which influence the initiation, triggering and termination of resident plans. Details of the ICI design are described elsewhere [14].

\section{GSR Data Fusion}

These concepts are currently being implemented and tested in the GSR, an experimental autonomous robot vehicle [1]. The GSR is a M114 armored personnel carrier which has been modified for computer control. It has sensors and processing for vehicle attitude, vision, navigation and 
proximity as well as computer control of all aspects of vehicle locomotion. An onboard planning subsystem is currently under development and a long range laser rangefinder is being added. With this sensor complement, the GSR provides a fertile testbed for sensor data fusion research. The underlying architecture permits data fusion to occur at a number of levels. While the blackboard is tree structured the GSR's functional structure is quite monolithic so any subsystem can interact with any other subsystem without regard for a architecturally imposed limitations. This implementation philosophy has provided a flexible framework within which to experiment with a variety of data fusion methods. Several examples of these are discussed below.

The vision subsystem is mounted on a three degree of freedom transport platform and will eventualiy incorporate both a solid state TV camera and the laser range finder for obstacle location, moving target tracking, terrain modelling, surface trafficability analysis and landmark navigation on the moving vehicle. Obviously, sensor data fusion will be necessary for these functions. For instance, camera and laser must interact to build the terrain map by using the contrast map obtained from the camera output to guide the discrete sampling of the computer steered laser rangefinder. Region range properties can be merged with relative depth cues from camera imagery to produce the terrain map. Trafficability estimates for different regions derived scene texture and laser range variability analyses can then be combined with this map for route planning purposes. Camera, laser and transport platform position data must be combined and merged to obtain landmark position to enhance vehicle absolute position estimates.

The proximity sensor subsystem uses acoustic ranging sensors to provide short range obstacle position and target tracking information. Some of these sensors are steered azimuthally to within a degree of accuracy while others are fixed to the robot. The proximity sensor subsystem must fuse the data from this array of nearly identical sensors into consistent target and obstacle position and velocity vectors. This is done largely using different averaging methods. Unfortunately, the acoustic ranging sensors have very poor angular resolution. One technique to overcome the problem inherent to these sensors is to use multiple sensors to triangulate upon the position of the target. This is another example of averaging methods for data fusion of information from like sensors. Further, during target tracking, vision estimates of target bearing can be fused with proximity estimates to improve the knowledge of target angular position and motion critical to accurate vehicle response. Likewise, coarse proximity information can be used to guide the camera steering and processing of the camera's complex visual information rapidly enough to permit timely vehicle control.

The vehicle attitude sensor subsystem monitors absolute vehicle speed, track speed, heading, pitch and roll angles. A fused value of vehicle speed is derived from a true ground speed sensor using doppler radar and a track speedometer using a confidence based decision method to take advantage of the greater low speed accuracy of the track speedometer yet use doppler speed for high speed or other times when track slippage conditions may prevail. Measurements of vehicle speed are combined with those of vehicle angular motion to estimate relative vehicle position. Then decision fusion methods are used to combine instantaneous vehicle position estimates from dead reckoning sensors with the intermittent absolute estimates from satellite and landmark navigation processes.

These are just a few examples of the sensor data fusion possibilities being explored on the GSR using the technique proposed in this paper.

\section{CONCLUSIONS}

While this technique has been implemented on an autonomous mobile robot it is general enough to be applied to any complex robot system or system of robots which must be implemented using distributed computing. The representation discussed above is flexible, is able to cope with a dynamic environment and supports the use of a number of different methods of data fusion. The computing architecture discussed above facilitates data fusion at a number of different levels by providing consistent access to system information.

While many different forms of sensor data fusion are currently implemented onboard the GSR many avenues of data fusion have not yet been explored. In the future, data fusion mechanisms will be further automated by employing the the data driven programming available through the active functions supported by the ICI. Through this capability appropriate data fusion methods can be switched dynamically. This hides higher level fusion from disinterested sensor subsystems thus further reducing complex robot design complexity.

\section{ACKNOWLEDGEMENTS}

The work discussed in this paper was supported jointly by the U. S. Marine Corps and the Naval Ocean Systems Center Internal Exploratory Development Program.

\section{REFERENCES}

[1] S. Harmon, "USMC Ground Surveillance Robot: A Testbed for Autonomous Vehicle Research", Proc. of the 4th UAH/UAB Robotics Conf., Huntsville, AL, Apr. 1984.

[2] J. W. Lowrie, M. Thomas, K. Gremban, M. Turk, "The Autonomous Land Vehicle (ALV) Preliminary Road Following Demonstration", Proc. of the SPIE Conf. on Computer Vision and Intelligent Robots, Cambridge, Mit, 1985. 
[3] P. Allen, R. Bajcsy, "Object Recognition Using Vision and Touch", Proc. of the 9th Int. Joint Conference on Artificial Intelligence, Los Angeles, CA, Aug. 1985, p1131-1135.

[4] E. Kent, T. Whe at ley, M. Nashman, "Real-time Cooperative Interaction between Structured-Light and Reflectance Ranging for Robot Guidance", Robotica, 3, 1985, p7-11.

[5] A. Flynn, Redundant Sensors for Mobile Robot Navigation, Dept. of Electrical Engineering and Computer Science, MIT, Cambridge, MA, July 1985.

[6] R. Brooks, A Robust Layered Control System for a Mobile Robot, A.I. Memo 864, MIT, Cambridge, MA, Sept. 1985.

[7] T. Iberal1, D. Lyons, Towards Perceptual Robotics, COINS T.R. 84-17, Laboratory for Perceptual Robotics, Univ. of Mass, Amherst, MA, Aug. 1984.

[8] M. Gini, et al, Symbolic Reasoning as a Basis for Automatic Error Recovery in Robots, Report 8511, Univ. of Minnesota, Minneapolis, MN, 1985.

[9] S. Harmon, "Autonomous Robot Submersibles: The Future of Unmanned Submersibles", Proc. of the 2nd ASME Computer Engineering Conf., San Diego, CA, Aug. 1982, p33-36.

[10] R. Brooks, "Visual Map Making for a Mobile Robot", Proc. of the IEEE Conf. on Robotics and Automation, St. Louis, MO, Mar. 1985, p824-829.

[11] J. Crowley, "Navigation of an Inte1ligent Mobile Robot", IEEE J. on Robotics and Automation, RA-1(1), Mar. 1985, p31-41.

[12] R. Chatila, J-P. Laumond, "Position Referencing and Consistent World Modelling for Mobile Robots", Proc. of the IEEE Conf. on Robotics and Automation, St. Louis, MO, Mar. 1985, p138-145.

[13] S. Harmon, "Coordination between Control and Knowledge Based Systems for Autonomous Vehicle Guidance", Proc. of the IEEE Trends and Applications Conf., Gaithersburg, MD, May 1983, p8-11.

[14] S. Harmon, D. Gage, W. Aviles, G. Bianchini, "Coordination of Intelligent Subsystems in Complex Robots", Proc. of the lst IEEE Conf. on Artificial Intelligence Applications, Denver, Co, Dec. 1984, p64-69. 